\begin{tabular}{|c|c|}
\hline Title & Characterization of HA CD $1 \mathrm{~K} 64 \mathrm{Q}$ mutant found in arrhythmogenic right ventricular dy splasia patients \\
\hline Author(s) & Konishi, Hiroyuki; Okuda, A yaka; Ohno, Y usuke; Kihara, A kio \\
\hline Citation & $\begin{array}{l}\text { Journal of Biochemistry, } 148(5), 617-622 \\
\text { https://doi.org/10.1093/jb/mvq092 }\end{array}$ \\
\hline Issue Date & 2010-11 \\
\hline Doc URL & http:/hdl.handle.net/2115/47347 \\
\hline Rights & $\begin{array}{l}\text { This is a pre copy-editing, author-produced PDF of an article accepted for publication in Journal of Biochemistry } \\
\text { following peer review. The definitive publisher-authenticated version } J \text { Biochem (2010) 148(5): 617-622 is available } \\
\text { online at: http://מb.oxfordj ournal s.org/content } / 48 / 5 / 617\end{array}$ \\
\hline Type & article (author version) \\
\hline File Information & JB1485_617-622.pdf \\
\hline
\end{tabular}

Instructions for use 
Regular Paper, Biochemistry

\title{
Characterization of HACD1 K64Q mutant found in arrhythmogenic right ventricular
}

\author{
dysplasia patients
}

\author{
Hiroyuki Konishi, Ayaka Okuda, Yusuke Ohno, and Akio Kihara* \\ Laboratory of Biochemistry, Faculty of Pharmaceutical Sciences, Hokkaido University, Kita 12-jo, \\ Nishi 6-choume, Kita-ku, Sapporo 060-0812, Japan
}

Running title: Characterization of HACD1 K64Q mutant

*Address correspondence to:

Akio Kihara

Laboratory of Biochemistry,

Faculty of Pharmaceutical Sciences, Hokkaido University,

Kita 12-jo, Nishi 6-choume, Kita-ku, Sapporo 060-0812, Japan

Telephone: +81-11-706-3754

Fax: +81-11-706-4900

E-mail: kihara@pharm.hokudai.ac.jp

Abbreviations: ARVD, arrhythmogenic right ventricular dysplasia; ER, endoplasmic reticulum; FAS, fatty acid synthase; SC, synthetic complete; VLCFA, very long-chain fatty acid. 


\begin{abstract}
Arrhythmogenic right ventricular dysplasia (ARVD) is an autosomal dominant heart disease. A K64Q mutation was found in ARVD-affected individuals in the HACD1 gene, which encodes an enzyme involved in very long-chain fatty acid (VLCFA) elongation, although any relationship between mutation and pathology remained unclear. Here, we demonstrate that HACD1 (K64Q) exhibits normal enzyme activity, intracellular localization, and interaction with other VLCFA enzymes, with no dominant negative effect on VLCFA elongation. Thus, it appears unlikely that this mutation is ARVD-causative. Moreover, through these analyses we found that HACD1 interacts with KAR and TER, the reductase enzymes involved in the second and fourth VLCFA elongation cycle, respectively. This finding indicates that the enzymes responsible for the VLCFA elongation cycle form an elongase complex.
\end{abstract}

Keywords: Arrhythmogenic right venticular dysplasia/ fatty acid/ heart/ lipid/ very long-chain fatty acid 


\section{Introduction}

Arrhythmogenic right ventricular dysplasia (ARVD) is an inherited heart disease characterized by a gradual loss of right ventricular myocardium and fibro-fatty infiltration with a prevalence of 1 in 2,500 to 1 in 5,000 $(1,2)$. It is a major cause of sudden death in young people and athletes $(1,2)$. To date, eight genes have been identified as being associated with ARVD: the DSP gene encoding desmoplaskin, PKP2 encoding plakophilin 2, DSC2 encoding desmocollin 2, DSG2 encoding desmoglein 2, JUP encoding plakoglobin, TGFB3 encoding transforming growth factor $\beta 3$, TMEM43 encoding transmembrane protein 43, and TP63 encoding tumor protein 63 (3). Most of these ARVD-causing mutations are autosomal dominant, although some autosomal recessive mutations have also been identified (2). Five of the ARVD genes encode desmosomal proteins (desmoplaskin, plakophilin 2, desmocollin 2, desmoglein 2, and plakoglobin) (1-3). Desmosome functions in cell adhesion, transcription regulation of genes involved in adipogenesis and apoptosis, regulation of gap junctions, and calcium homeostasis (2). The ARVD mutations demonstrate incomplete penetrance and variable expressivity $(1,2)$, suggesting that environmental factors and differences in genetic background are also involved in the pathogenesis.

Several linkage analyses have identified ARVD-associated loci differing from the above genes (2). One such locus was mapped at 10p12-14 with ARVD segregating as an autosomal, dominant transmission (4). The HACD1 (PTPLA) gene is located in this region, and a point mutation known to cause a K64Q substitution in the HACD1 protein was found in all the affected individuals of a large ARVD family ( $\mathrm{n}=10)$ (5). However, this mutation was also detected in normal control subjects (3 alleles per 100 chromosomes) (5). Incomplete penetrance of ARVD has impeded conclusions as to whether this mutation causes ARVD, making the enzymatic characterization of HACD1 (K64Q) essential for the determination. Until recently, however, even the function of HACD1 had not been 
known. In 2008, though, we identified HACD1 as the 3-hydroxyacyl-CoA dehydratase involved in elongation of very long-chain fatty acids (VLCFAs) (6). This work enabled us to perform biochemical analyses of an HACD1 (K64Q) mutant protein.

VLCFAs are fatty acids with a chain-length of $\geq 20$ that function in numerous cellular processes, including sphingolipid biogenesis, inflammation, immunity, fetal growth and development, retinal function, and brain development (7-9). VLCFA elongation occurs by cycling through a 4 step process (condensation, reduction, dehydration, and reduction), with 2 carbons added through each cycle. The first condensation step is catalyzed by one of seven elongases (ELOVL1-7) $(8,9)$. The second and fourth reduction steps are catalyzed by the reductases KAR and TER, respectively (10).

HACD1/PTPLA and three homologous proteins (HACD2/PTPLB, HACD3/PTPLAd1, and HACD4/PTPLAd2) are responsible for the third step, catalyzing the dehydration of 3-hydroxyacyl-CoA (6). HACD1 mRNA is expressed in heart at high levels and is also found in skeletal muscle, but virtually no expression has been detected in other tissues tested (5). The high level expression of HACD1 mRNA in heart is in strong accordance with the concept that HACD1 is ARVD-causative gene.

In the presented study we performed an enzymatic characterization of the mutant HACD1 (K64Q). HACD1 (K64Q) exhibited 3-hydroxyacyl-CoA dehydratase activity at levels indistinguishable from wild type HACD1. In addition, ectopic expression of HACD1 (K64Q) was able to restore the growth of a yeast mutant defective in 3-hydroxyacyl-CoA dehydratase (Phs1). Moreover, HACD1 (K64Q) localized normally in the ER and interacted with other components of the VLCFA elongation machinery. Finally, we could not find any dominant negative effects of HACD1 (K64Q) on VLCFA elongation when overproduced in HEK 293T cells or in C2C12 myoblasts. All these results suggest that the HACD1 (K64Q) mutation is not responsible for ARVD pathology. 


\section{Materials and Methods}

\section{Cell culture and transfection}

HEK 293 T and C2C12 cells were grown in Dulbecco’s modified Eagle’s medium (Sigma, St. Louis, MO) containing $10 \%$ fetal bovine serum and supplemented with 100 units/ml penicillin and $100 \mu \mathrm{g} / \mathrm{ml}$ streptomycin. The C2C12 myoblasts were provided by the RIKEN BRC through the National Bio-Resource Project of the MEXT, Japan. HEK 293T cells were grown in 0.3\% collagen-coated dishes. Transfections were performed using Lipofectamine Plus ${ }^{\mathrm{TM}}$ Reagent (Invitrogen, Carlsbad, CA) according to the manufacturer’s instructions.

S. cerevisiae strains R1158 and TH_3237 (pPHS1::KanMX4-TetO-CYC TATA $_{7}$ (11) were obtained from Open Biosystems (Huntsville, AL). Cells were grown in synthetic complete (SC) medium (0.67\% yeast nitrogen base and 2\% D-glucose) containing nutritional supplements.

\section{Plasmids}

The pCE-puro 3xFLAG-1, pCE-puro HA-1, and the pCE-puro His 6 -Myc-1 plasmids are mammalian expression vectors and were designed to produce an N-terminal 3xFLAG-tagged protein, an N-terminal HA-tagged protein, and an N-terminal tandemly oriented $\mathrm{His}_{6}$ and Myc epitope (His 6 -Myc)-tagged protein, respectively. The pCE-puro 3xFLAG-HACD1, pCE-puro His $_{6}$-Myc-HACD1, and pCE-puro HA-ELOVLx (where $\mathrm{x}$ is each ELOVL number) plasmids have been described previously (6). The pAKNF313 plasmid (HIS3 marker) is a yeast expression vector and was designed to produce an N-terminal 3xFLAG-tagged protein.

The KAR and TER genes were amplified from an EST clone (ID 3622879) (Open Biosystems, Huntsville, AL) and from human liver cDNA (Clontech (TAKARA Bio), Palo Alto, CA), respectively, by PCR using primers (for KAR, 5'-AGGATCCATGGAGAGCGCTCTCCCCGCCGCCG-3' (BamHI 
site underlined) and 5'-TTTAGTTCTTCTTGGTTTTCTTCAGATAG-3’; and for TER, 5'-AGGATCCATGAAGCATTACGAGGTGGAGATTCTG-3' (BamHI site underlined) and 5’-TTCAGAGCAGGAAGGGGATGATGGGC-3’). The resulting fragments were first cloned into pGEM-T Easy vector (Promega, Madison, WI), generating the pGEM-KAR and pGEM-TER plasmids. The pCE-puro 3xFLAG-KAR and pCE-puro 3xFLAG-TER plasmids were constructed by cloning the BamHI-NotI fragments of each of the pGEM-KAR and pGEM-TER plasmids into a pCE-puro 3xFLAG-1 vector.

The K64Q mutant of HACD1 was constructed by site-directed mutagenesis using a QuikChange site-directed mutagenesis kit (Stratagene, Agilent Technologies, La Jolla, CA). The sequences of the primers used were: 5'-GGAGGCTCCCGGCCAGCGGAGGCGCCTGG-3’ and 5'-CCAGGCGCCTCCGCTGGCCGGGAGCCTCC-3'. The HACD1 and HACD1(K64Q) genes were cloned into the pAKNF313 plasmid to generate the pKH10 and pKH11 plasmids, respectively.

\section{Immunological Assays}

Co-immunoprecipitation experiments were performed as described previously (12), using the anti-Myc antibody PL14 (Medical \& Biological Laboratories, Nagoya, Japan). Immunoblotting was performed as described previously (13) using the anti-FLAG antibody M2 (1 $\mu \mathrm{g} / \mathrm{ml})$, the anti-HA antibody HA-7 (1:2000 dilution; Sigma), or the anti-Myc antibody PL14 (0.5 $\mu \mathrm{g} / \mathrm{ml})$ as the primary antibody and HRP-conjugated anti-mouse IgG F(ab’) $)_{2}$ fragment (from GE Healthcare Bio-Sciences, Piscataway, NJ, and diluted 1:7500) as the secondary antibody. Labeling was detected using ECL ${ }^{\mathrm{TM}}$ Reagents or an ECL plus System for Western Blotting Detection (both from GE Healthcare Bio-Sciences). Microscopic immunofluorescence studies were performed with the anti-Myc antibody PL14 (5 $\mu \mathrm{g} / \mathrm{ml}$ ) and an anti-calreticulin antibody (5 $\mu \mathrm{g} / \mathrm{ml}$; Alexis, Lausen, Switzerland) (6). 


\section{In Vitro 3-Hydroxyacyl-CoA Dehydratase Assays}

In vitro 3-hydroxyacyl-CoA dehydratase assays were performed as described previously (6). Using anti-FLAG M2 agarose (Sigma), 3xFLAG-tagged HACD1 proteins were purified from HEK 293T cells transfected with the pCE-puro 3xFLAG-HACD1 or pCE-puro 3xFLAG-HACD1 (K64Q) plasmid. In vitro 3-hydroxyacyl-CoA dehydratase assays were performed by mixing purified proteins in reaction buffer (total volume of $40 \mu \mathrm{l} ; 50 \mathrm{mM}$ Tris- $\mathrm{HCl}$ (pH 7.5), $150 \mathrm{mM} \mathrm{NaCl}, 10 \%$ glycerol, 1 $\mathrm{mM}$ dithiothreitol, $1 \mathrm{mM}$ phenylmethylsulfonyl fluoride, and $1 \mathrm{X}$ protease inhibitor mixture (Complete ${ }^{\mathrm{TM}}$ EDTA free; Roche Diagnostics, Indianapolis, IN), 0.1\% Tritin X-100, and $1 \mathrm{mM} \mathrm{MgCl}_{2}$ ) with $0.01 \mu \mathrm{Ci}\left[{ }^{14} \mathrm{C}\right] 3$-hydroxypalmitoyl-CoA (55 mCi/mmol; American Radiolabeled Chemicals, St. Louis, MO), then incubating the mixture at $37^{\circ} \mathrm{C}$ for 10 or $30 \mathrm{mn}$. The reactions were terminated by adding $20 \mu \mathrm{l} 75 \% \mathrm{KOH}(\mathrm{w} / \mathrm{v})$ and $40 \mu \mathrm{l}$ ethanol, then saponified at $70^{\circ} \mathrm{C}$ for $1 \mathrm{~h}$, and acidified by adding $80 \mu \mathrm{l} 5 \mathrm{~N} \mathrm{HCl}$ with $40 \mu \mathrm{l}$ ethanol. Lipids were extracted twice, each with $700 \mu \mathrm{l}$ hexane, then the extracts were pooled, dried, and suspended in $35 \mu \mathrm{l}$ chloroform. Lipids were separated by TLC on LK50F Silica Gel 150A TLC plates (Whatman, Kent, UK) with hexane/diethyl ether/acetic acid (30:70:1, v/v) as the solvent system. The radioactivities associated with the reaction product 2,3-trans hexadecenoic acid were quantified using a bioimaging analyzer BAS-2500 (Fuji Photo Film, Tokyo, Japan).

\section{In Vitro Fatty Acid Elongation Assays}

In vitro fatty acid elongation assays were performed essentially as described elsewhere (14) using total membrane fractions. Cells were suspended in buffer A (50 mM HEPES-NaOH (pH 6.8), 10\% glycerol, $150 \mathrm{mM} \mathrm{NaCl}$, $1 \mathrm{X}$ protease inhibitor mixture, $1 \mathrm{mM}$ dithiothreitol, and $1 \mathrm{mM}$ 
phenylmethylsulfonyl fluoride) and lysed by sonication. After ultracentrifugation (100,000 g, 30 min, $4{ }^{\circ} \mathrm{C}$ ), the pellet was suspended in buffer A and was used as the total membrane fraction. Typical reaction mixtures of fatty acid elongation assays contained total membrane fractions (20 $\mu$ g protein), $50 \mu \mathrm{M}$ palmitoyl-CoA complexed with $0.2 \mathrm{mg} / \mathrm{ml}$ fatty acid-free bovine serum albumin (Sigma), and $0.025 \mu \mathrm{Ci}\left[{ }^{14} \mathrm{C}\right]$ malonyl-CoA (55 mCi/mmol; Moravek Biochemicals, Brea, CA) in a $50 \mu$ l reaction mixture (buffer A containing $2 \mathrm{mM} \mathrm{MgCl} 2,1 \mathrm{mM} \mathrm{CaCl}_{2}$, and $1 \mathrm{mM} \mathrm{NADPH}$ ). After a $30 \mathrm{~min}$ incubation at $37^{\circ} \mathrm{C}$, the reactions were terminated by adding $25 \mu \mathrm{l} 75 \% \mathrm{KOH}(\mathrm{w} / \mathrm{v})$ and $50 \mu \mathrm{l}$ ethanol. Lipids were saponified, acidified, extracted, and separated by normal phase TLC as described above. 


\section{Results}

\section{HACD1 (K64Q) exhibits normal 3-hydroxyacyl-CoA dehydratase activity}

HACD1 was recently identified as a 3-hydroxyacyl-CoA dehydratase in mammals (6). To examine and compare their enzyme activities, the mutant HACD1 (K64Q) and wild type HACD1 were expressed in HEK 293T cells as N-terminally 3xFLAG tagged proteins. The proteins were affinity-purified with anti-FLAG antibodies then subjected to an in vitro 3-hydroxyacyl-CoA dehydratase assay using $\left[{ }^{14} \mathrm{C}\right] 3$-hydroxypalmitoyl-CoA as a substrate. The wild type 3xFLAG-HACD1 protein exhibited dehydratase activity in a time-dependent manner (Fig. 1A). Moreover, the 3xFLAG-HACD1 (K64Q) protein exhibited activity at levels similar to those of the wild type protein (Fig. 1A).

HACD1 is conserved among eukaryotes, and yeast have an HACD1 homolog called Phs1 (15). Since the production of VLCFAs in yeast is essential for growth, deletion of the PHS1 gene is lethal (11). Using yeast cells in which expression of the PHS1 mRNA is under the control of a tetracycline-dependent promoter ( $\mathrm{TetO}_{7}-\mathrm{PHS1}$ cells), we previously demonstrated that the expression of wild type HACD1 could restore the deficient growth in the presence of a tetracycline antibiotic (6). In the presented study, we introduced the HACD1 (K64Q) gene into the TetO $O_{7}-P H S 1$ cells and examined its activity to complement cell growth. Similar to results with the wild type HACD1 gene, the HACD1 (K64Q) gene also restored the growth of the $\mathrm{TetO}_{7}-\mathrm{PHS1}$ cells in the presence of the antibiotic (Fig. 1B). This result confirms the conclusion drawn from the above result that HACD1 (K64Q) is an active enzyme.

\section{HACD1 (K64Q) is localized in the ER}

To fulfill its own function in vivo, each protein must be localized in the proper organelle. 
However, certain mutations can cause mislocalization of the resulting protein. Such mislocalization-inducing mutations are indeed known in another VLCFA-related gene, the elongase ELOVL4. Since VLCFA synthesis occurs in the endoplasmic reticulum (ER), all components of the VLCFA elongation cycle are normally localized in the ER. However, mutations near the 3' terminus of the ELOVL4 coding sequence cause a truncation of the C-terminus of ELOVL4, resulting in the mislocalization of the mutated protein and the development of autosomal dominant Stargardt-like macular dystrophy (16). With this in mind, we next examined the possibility that the K64Q mutation affects the intracellular localization of HACD1 using C2C12 myoblasts. Lys-64 had been predicted to be a part of a nuclear localization signal, although the intracellular localization of wild type or the K64Q mutant was not investigated in that report (5). In our studies here, indirect immunofluorescence microscopy demonstrated that the wild type $\mathrm{His}_{6}$-Myc tagged HACD1 protein was localized in the ER, as illustrated by the co-localization of the ER marker calreticulin (Fig. 2). The HACD1 (K64Q) mutant was also localized in the ER and similarly merged with calreticulin (Fig. 2). Therefore, HACD1 is an ER-resident protein, and the K64Q mutation did not disturb its localization.

\section{HACD1 (K64Q) interacts normally with other components of the VLCFA elongation machinery}

In yeast, enzymes involved in the VLCFA elongation cycle form an elongase complex (15). In our previous study we revealed that HACD1 interacts with the elongases involved in this complex (ELOVL1-7) (6). Here, we examined whether HACD1 also interacts with other components of the VLCFA elongation machinery, specifically the reductases KAR and TER, which are responsible for the second and fourth steps in the VLCFA elongation, respectively. We conducted co-immunoprecipitation experiments using anti-Myc antibodies and lysates prepared from HEK 293T cells expressing combinations of a $\mathrm{His}_{6}$-Myc -tagged wild type HACD1 and each FLAG-tagged 
reductase protein. We found that wild type HACD1 interacts with both KAR (Fig. 3A) and TER (Fig. 3B), indicating that mammalian proteins also form a VLCFA elongase complex.

As described above, HACD1 (K64Q) exhibited normal enzyme activity and localization. However, it is possible that interaction with other components of the VLCFA elongase complex is impaired by the K64Q mutation, resulting in inefficient VLCFA production. Therefore, we investigated the interactions of HACD1 (K64Q) with the other components of the VLCFA elongase complex by co-immunoprecipitation experiments. Our results indicate that HACD1 (K64Q) interacts with each of the ELOVL elongases ELOVL1-7 (Fig. 3C), and with KAR (Fig. 3A) or TER (Fig. 3B), as efficiently as the wild type HACD1 protein. Thus, the K64Q mutation does not impair the formation of the elongase complex.

\section{HACD1 (K64Q) does not exhibit any dominant negative effects on VLCFA elongation}

The mutation in the 10p12-14 chromosomal locus associated with ARVD is segregated as an autosomal dominant transmission (4). Therefore, it was expected that if the K64Q mutation is ARVD-causative, expression of the HACD1 (K64Q) protein would exhibit a dominant negative effect on VLCFA elongation. To test this possibility, we performed an in vitro fatty acid elongation assay, in which total membrane fractions prepared from HEK $293 \mathrm{~T}$ cells transfected with the vector or plasmid encoding wild type HACD1 or HACD1 (K64Q) were incubated with palmitoyl-CoA and $\left[{ }^{14} \mathrm{C}\right]$ malonyl-CoA in the presence of NADPH. Control membranes converted palmitoyl-CoA to acyl-CoAs (mainly stearoyl-CoA) via 3-keto, 3-hydroxy-, and 2,3-trans-enoyl acyl-CoA intermediates (Fig. 4). Overproduction of wild type HACD1 did not cause any significant change in the amounts of the acyl-CoA products, consistent with the theory that the step catalyzed by HACD1 is not rate-limiting (Fig. 4). The expression of HACD1 (K64Q) also did not affect the product levels (Fig. 4). 
Similar results were obtained when C2C12 cells were used instead of HEK 293T cells (Fig. 4). These results indicate that the HACD1 (K64Q) protein exhibits no dominant negative effect in regards to VLCFA elongation. 


\section{Discussion}

ARVD is a hereditary cardiomyopathy characterized by replacement of myoblasts with fibrofatty tissue, ventricular tachyarrythmias, and sudden death $(1,2)$. Although eight genes have been identified as ARVD-causative genes, including many that encode components of the cardiac desmosome, some ARVD-associated chromosomal loci have yet to be associated with their responsible genes (2, 3). The HACD1 gene is located in one such locus, 10p12-14, and previous results had demonstrated that ARVD patients carry a point mutation that causes K64Q substitution in the HACD1 protein (5). However, this mutation was also found in normal controls at low frequency (5). The incomplete penetrance nature of ARVD has made it difficult to interpret whether this mutation is responsible for the ARVD pathology. Therefore, enzymatic characterization of HACD1 (K64Q) was needed.

In the presented study, we examined the enzyme activity of HACD1 (K64Q) and found that it exhibited a 3-hydroxyacyl-CoA dehydratase activity comparable to that of the wild type HACD1 protein (Fig. 1A). Expression of HACD1 (K64Q) in the TetO ${ }_{7}-P H S 1$ cells restored their deficient growth (Fig. 1B). Moreover, HACD1 (K64Q) was properly localized in the ER (Fig. 2) and was able to interact with other components of the VLCFA elongase complex, similar to wild type HACD1 (Fig. 3). All these results indicate that HACD1 (K64Q) exhibits enzymatic properties indistinguishable from wild type HACD1.

HACD1 is conserved among eukaryotes, and together the homologs constitute the Phs1 family. We previously examined the membrane topology of the yeast Phs1 protein and determined it to be a six-span membrane protein with its N-terminus and C-terminus both facing toward the cytosol (11). When amino acid sequences of 31 Phs1 family members from 24 organisms were compared, 22 amino acids were found to be conserved (11). By mutating each of the 22, we determined that six 
amino acids are important in Phs1 function, and, of these, two are indispensable (11). The six important residues are each located within or near transmembrane regions 3 and 5 (11). Lys-64 of HACD1 is located in the first N-terminal hydrophilic domain. This residue is not conserved among Phs1 family members. Furthermore, the lengths of the N-terminal hydrophilic domains vary among Phs1 family members, with the proteins found in lower eukaryotes having shorter N-terminal hydrophilic domains; these domains do not have the residue corresponding to Lys-64 of human HACD1. The low evolutionary conservativeness of this residue is consistent with our findings that Lys-64 of HACD1 was dispensable for HACD1 function.

The ARVD mutation associated with 10p12-14 segregates in an autosomal dominant fashion, as do most of the other ARVD mutations (4). Therefore, we investigated whether HACD1 (K64Q) exhibited any dominant negative effects on VLCFA elongation. However, no such effects were observed for HACD1 (K64Q) when overproduced in HEK 293T cells or C2C12 myoblasts (Fig. 4). All these results strongly suggest that the HACD1 (K64Q) mutation is not responsible for the ARVD pathology.

The HACD1 gene may not be responsible for the ARVD pathology, but there could be others not yet recognized. One candidate gene is CACNB2, located at 10p12 near the HACD1 gene. The CACNB2 gene encodes the $\beta_{2 b}$ subunit of the L-type calcium channel. A recent study reported that a loss-of-function mutation in this gene is associated with a familial sudden cardiac death syndrome with ST-segment elevation and short QT intervals (17). Although the electrocardiogram abnormalities associated with the reported ARVD mutation at 10p12-14 would seem not to correlate with the CACNB2-associated pathology, it is possible that mutational differences could cause such phenotypic differences.

An insertion mutation in the $H A C D 1$ gene causes an autosomal recessive centronuclear 
myopathy in dogs (18). Although skeletal muscle atrophy is observed in this disease, abnormalities in heart have not been reported. HACD1 is highly expressed in heart, suggesting that it plays unknown functions in that organ.

We recently reported that HACD proteins interact with ELOVLs (6). In the presented study, we demonstrated that HACD1 also interacts with the reductases KAR and TER (Fig. 3). Thus, all components of VLCFA elongation form an elongase complex. These interactions had already been established for the yeast VLCFA elongation components, so it would appear that the VLCFA elongase complexes are evolutionally conserved. Mammalian fatty acid synthase (FAS), which produces palmitic acid, is a multi-enzyme and incorporates all catalytic activities of its cyclic reaction as discrete domains on one polypeptide chain (19). Like FAS, the adjacent positioning of the catalytic centers of the four elongation reactions of VLCFA elongation may contribute to efficient cycling. However, future in vitro studies are needed for elucidation of the molecular mechanism of the presumptive cooperative progression in the VLCFA elongation cycle. 


\section{Acknowledgements}

We are grateful to Dr. E. A. Sweeney for scientific editing of the manuscript. This work was supported by a Grant-in-Aid for Young Scientists (A) (20687008) from the Ministry of Education, Culture, Sports, Sciences and Technology of Japan and in part by grants from the ONO Medical Research Foundation. 


\section{References}

1. Thiene, G., Corrado, D., and Basso, C. (2007) Arrhythmogenic right ventricular cardiomyopathy/dysplasia. Orphanet J. Rare Dis. 2, 45

2. Awad, M.M., Calkins, H., and Judge, D.P. (2008) Mechanisms of disease: molecular genetics of arrhythmogenic right ventricular dysplasia/cardiomyopathy. Nat. Clin. Pract. Cardiovasc. Med. 5, 258-267

3. van der Zwaag, P.A., Jongbloed, J.D., van den Berg, M.P., van der Smagt, J.J., Jongbloed, R., Bikker, H., Hofstra, R.M., and van Tintelen, J.P. (2009) A genetic variants database for arrhythmogenic right ventricular dysplasia/cardiomyopathy. Hum. Mutat. 30, 1278-1283

4. Li, D., Ahmad, F., Gardner, M.J., Weilbaecher, D., Hill, R., Karibe, A., Gonzalez, O., Tapscott, T., Sharratt, G.P., Bachinski, L.L., and Roberts, R. (2000) The locus of a novel gene responsible for arrhythmogenic right-ventricular dysplasia characterized by early onset and high penetrance maps to chromosome 10p12-p14. Am. J. Hum. Genet. 66, 148-156

5. Li, D., Gonzalez, O., Bachinski, L.L., and Roberts, R. (2000) Human protein tyrosine phosphatase-like gene: expression profile, genomic structure, and mutation analysis in families with ARVD. Gene 256, 237-243

6. Ikeda, M., Kanao, Y., Yamanaka, M., Sakuraba, H., Mizutani, Y., Igarashi, Y., and Kihara, A. (2008) Characterization of four mammalian 3-hydroxyacyl-CoA dehydratases involved in very long-chain fatty acid synthesis. FEBS Lett. 582, 2435-2440

7. Spector, A.A. (1999) Essentiality of fatty acids. Lipids 34 Suppl, S1-S3

8. Leonard, A.E., Pereira, S.L., Sprecher, H., and Huang, Y.S. (2004) Elongation of long-chain fatty acids. Prog. Lipid Res. 43, 36-54

9. Jakobsson, A., Westerberg, R., and Jacobsson, A. (2006) Fatty acid elongases in mammals: 
their regulation and roles in metabolism. Prog. Lipid Res. 45, 237-249

10. Moon, Y.A., and Horton, J.D. (2003) Identification of two mammalian reductases involved in the two-carbon fatty acyl elongation cascade. J. Biol. Chem. 278, 7335-7343

11. Kihara, A., Sakuraba, H., Ikeda, M., Denpoh, A., and Igarashi, Y. (2008) Membrane topology and essential amino acid residues of Phs1, a 3-hydroxyacyl-CoA dehydratase involved in very long-chain fatty acid elongation. J. Biol. Chem. 283, 11199-11209

12. Kihara, A., Anada, Y., and Igarashi, Y. (2006) Mouse sphingosine kinase isoforms SPHK1a and SPHK1b differ in enzymatic traits including stability, localization, modification, and oligomerization. J. Biol. Chem. 281, 4532-4539

13. Kihara, A., Ikeda, M., Kariya, Y., Lee, E.Y., Lee, Y.M., and Igarashi, Y. (2003)

Sphingosine-1-phosphate lyase is involved in the differentiation of F9 embryonal carcinoma cells to primitive endoderm. J. Biol. Chem. 278, 14578-14585

14. Moon, Y.A., Shah, N.A., Mohapatra, S., Warrington, J.A., and Horton, J.D. (2001) Identification of a mammalian long chain fatty acyl elongase regulated by sterol regulatory element-binding proteins. J. Biol. Chem. 276, 45358-45366

15. Denic, V., and Weissman, J.S. (2007) A molecular caliper mechanism for determining very long-chain fatty acid length. Cell 130, 663-677

16. Karan, G., Yang, Z., and Zhang, K. (2004) Expression of wild type and mutant ELOVL4 in cell culture: subcellular localization and cell viability. Mol. Vis. 10, 248-253

17. Antzelevitch, C., Pollevick, G.D., Cordeiro, J.M., Casis, O., Sanguinetti, M.C., Aizawa, Y., Guerchicoff, A., Pfeiffer, R., Oliva, A., Wollnik, B., Gelber, P., Bonaros, E.P., Jr., Burashnikov, E., Wu, Y., Sargent, J.D., Schickel, S., Oberheiden, R., Bhatia, A., Hsu, L.F., Haissaguerre, M., Schimpf, R., Borggrefe, M., and Wolpert, C. (2007) Loss-of-function mutations in the cardiac 
calcium channel underlie a new clinical entity characterized by ST-segment elevation, short QT intervals, and sudden cardiac death. Circulation 115, 442-449

18. Pelé, M., Tiret, L., Kessler, J.L., Blot, S., and Panthier, J.J. (2005) SINE exonic insertion in the PTPLA gene leads to multiple splicing defects and segregates with the autosomal recessive centronuclear myopathy in dogs. Hum. Mol. Genet. 14, 1417-1427

19. Smith, S. (1994) The animal fatty acid synthase: one gene, one polypeptide, seven enzymes. FASEB J. 8, 1248-1259 


\section{Figure legends}

Fig. 1. The mutant HACD1 (K64Q) exhibits normal 3-hydroxyacyl-CoA dehydratase activity. (A) HEK $293 \mathrm{~T}$ cells were transfected with a vector (pCE-puro 3xFLAG-1) or a plasmid encoding the wild type or K64Q mutant of HACD1. The HACD1 proteins were affinity-purified from their lysates using anti-FLAG M2 beads, and samples (2 ng protein) were incubated with $4.5 \mu \mathrm{M}$ $\left[{ }^{14} \mathrm{C}\right] 3$-hydroxypalmitoyl-CoA for 10 or $30 \mathrm{~min}$ at $3 \mathrm{C}$. After termination of the reactions, lipids were saponified, acidified, extracted, and separated by TLC. The radioactivities associated with the reaction product 2,3-trans hexadecenoic acid were quantified using a bioimaging analyzer BAS-2500. Values represent the mean \pm S.D. from three independent experiments. (B) R1158 (wild type; WT) and TH_3237 (TetO-PHS1; Tet) cells were transfected with the pAKNF313 (vector), pKH10 (3xFLAG-HACD1), or pKH11 (3xFLAG-HACD1 (K64Q)) plasmid. Cells were grown for 2 days at 30 ${ }^{\circ} \mathrm{C}$ on SC plates lacking histidine but containing $10 \mu \mathrm{g} / \mathrm{ml}$ doxycycline, a tetracycline antibiotic.

Fig. 2. HACD1 (K64Q) is localized in the ER. C2C12 cells were transfected with a plasmid encoding the wild type or K64Q mutant of HACD1. The transfected cells were fixed with formaldehyde and permeabilized with $0.1 \%$ Triton X-100. The cells were then double stained with anti-Myc (left panels) and anti-calreticulin (middle panels) antibodies. Fluorescent images were obtained using a confocal laser scanning microscope (LSM510 META; Carl Zeiss, Oberkochen, Germany). Merged images are presented in the right panels. Calibration bar, $10 \mu \mathrm{m}$.

Fig. 3. HACD1 (K64Q) interacts with the components of the VLCFA elongation cycle.

HEK 293T cells were transfected with a pCE-puro His ${ }_{6}$-Myc-1 (vector), pCE-puro His 6 -Myc-HACD1, or pCE-puro His ${ }_{6}$-Myc-HACD1 (K64Q) plasmid and with the pCE-puro 3xFLAG-KAR (A), 
pCE-puro 3xFLAG-TER (B), or pCE-puro HA-ELOVLx (x represents each ELOVL number) (C) plasmid. Total cell lysates were prepared from the transfected cells and solubilized with $1 \%$ Triton X-100. Following immunoprecipitation with the anti-Myc antibody PL14, total lysates and bound proteins were subjected to immunoblotting with anti-FLAG, anti-Myc, or anti-HA antibodies. Bound proteins were loaded at 5 times the amount of total lysates. IP, immunoprecipitation; IB, immunoblotting; V, vector; W, wild type; M, mutant (K64Q).

Fig. 4. HACD1 (K64Q) does not exhibit any dominant negative effects on VLCFA synthesis. HEK 293T cells and C2C12 myoblasts were transfected with a pCE-puro 3xFLAG-1 (vector), pCE-puro 3xFLAG-HACD1, or pCE-puro 3xFLAG-HACD1 (K64Q) plasmid. Total membrane proteins (20 $\mu$ g protein) were prepared from the transfected cells and were incubated with palmitoyl-CoA $(50 \mu \mathrm{M})$ and $0.025 \mu \mathrm{Ci}\left[{ }^{14} \mathrm{C}\right]$ malonyl-CoA $(9.1 \mu \mathrm{M})$ in the presence of $1 \mathrm{mM}$ NADPH, for $30 \mathrm{~min}$ at $37^{\circ} \mathrm{C}$. After termination of the reactions, lipids were saponified, acidified, extracted, and separated by normal phase TLC. The radioactivities associated with the reaction product fatty acids were quantified using a bioimaging analyzer BAS-2500. Values represent the mean \pm S.D. from three independent experiments. 
A

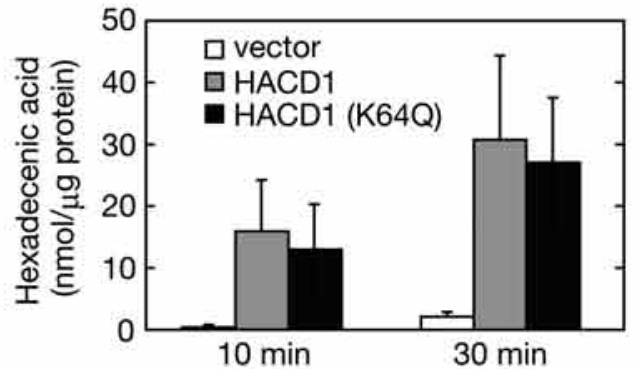

B

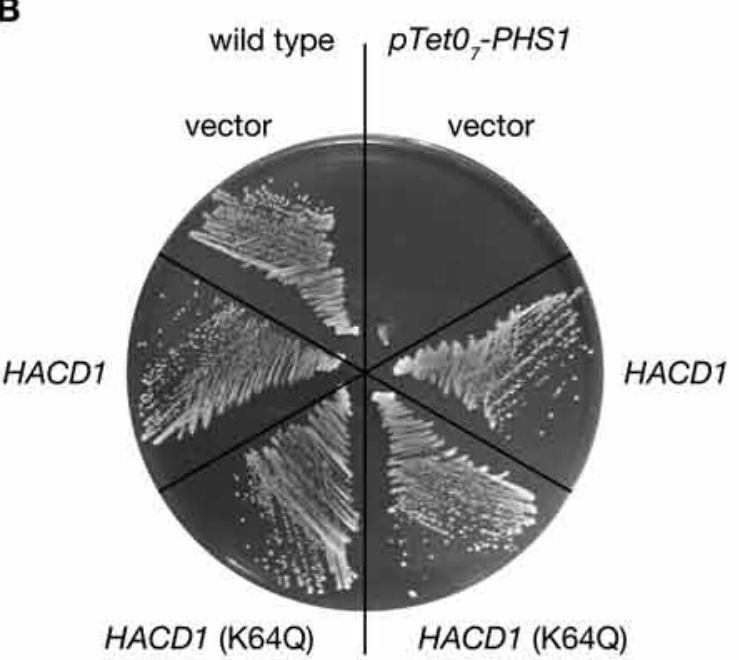

Konishi et al., Fig. 1 


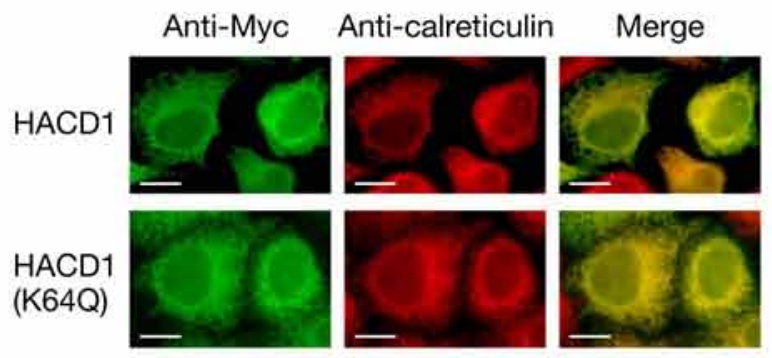

Konishi et al., Fig. 2 


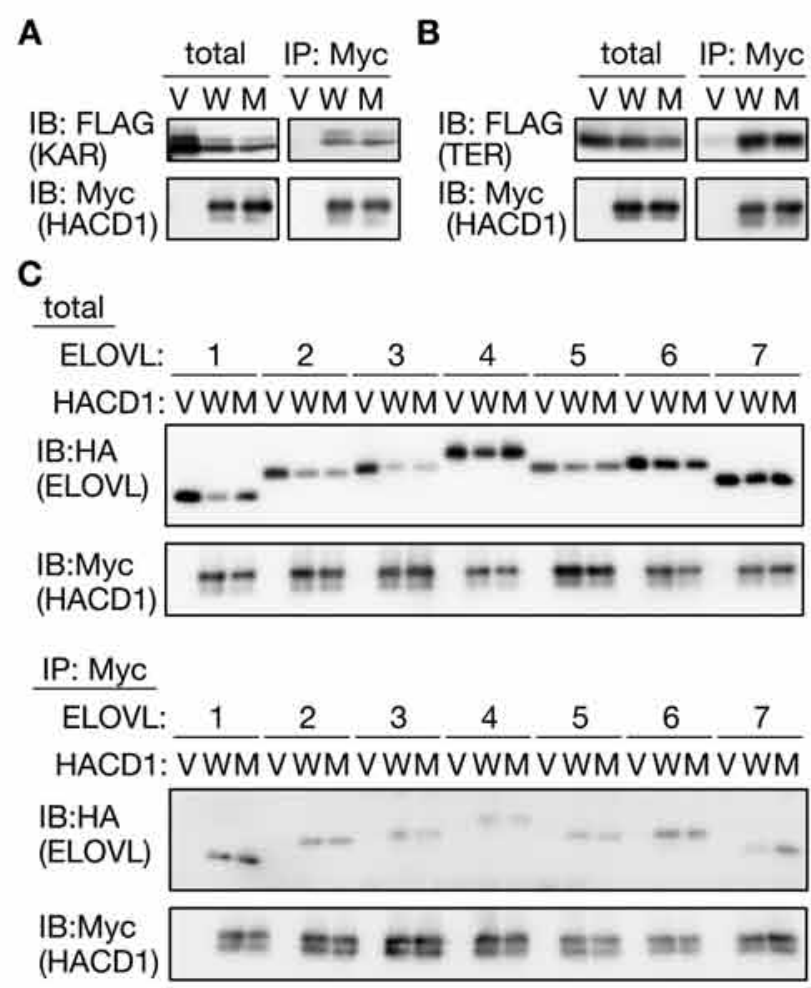

Konishi et al., Fig. 3 


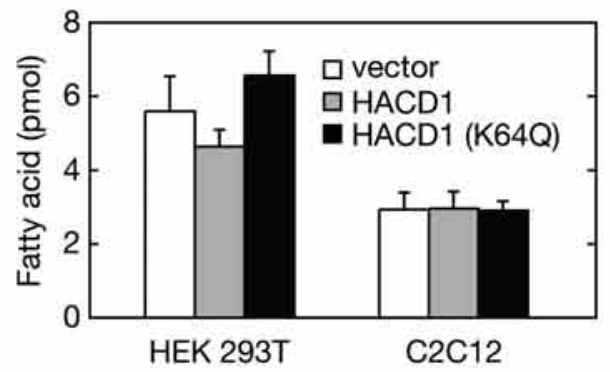

Konishi et al., Fig. 4 